\title{
TRACING THE INTERSTELLAR MEDIUM IN OPHIUCHUS ACROSS 14 ORDERS OF MAGNITUDE IN FREQUENCY
}

\author{
S.W. DIGEL ${ }^{1,2}$, S.D. HUNTER ${ }^{2}$ AND S.L. SNOWDEN ${ }^{2,3}$ \\ ${ }^{1}$ Hughes STX \\ ${ }^{2}$ NASA Goddard Space Flight Center \\ ${ }^{3}$ Universities Space Research Association
}

\section{Introduction}

The spectral range of ground and space-based observations has expanded rapidly in recent years. Essentially complete sky coverage is available in bands spanning more than 14 orders of magnitude in frequency, with varying sensitivities and resolutions. Remarkably, extended emission (and absorption) associated with the interstellar medium can be seen across this range. This poster illustrated the range of information now at hand for just a single interstellar cloud complex, that in Ophiuchus. This complex, well known for its abundant on-going star formation, is relatively nearby $(\sim 125$ pc), and well removed from the plane, offering the advantages of large angular size and little confusion in most bands from background Galactic emission.

TABLE 1. Data Sources

\begin{tabular}{lll}
\hline Image & Bands & Reference \\
\hline H I & $21 \mathrm{~cm}$ & Burton 1985; Digel et al. 1992, unpub. \\
CO & $2.6 \mathrm{~mm}$ & de Geus et al. 1990 \\
IRAS & $12,60,100 \mu \mathrm{m}$ & Wheelock et al. 1994 \\
DIRBE & $1.2,2.2,3.5 \mu \mathrm{m}$ & Hauser et al. 1995 \\
DSS & $0.6 \mu \mathrm{m}$ & Postman et al., in preparation \\
ROSAT & $0.75,1.5 \mathrm{keV}$ & Snowden et al. 1994 \\
EGRET & $>100 \mathrm{MeV}$ & Hunter et al. 1994; Digel \& Hunter 1994 \\
\hline
\end{tabular}


Owing to the limited space available, only a sample of the images from various bands for the central part of the Ophiuchus complex are presented here (Figure 1); for descriptions of the observations and the analyses, the reader is referred to the references cited in Table 1. Analyses involving more than one band have been used to determine the spatial distributions, column densities, and temperatures of the interstellar gas and dust in Ophiuchus, as well as the line-of-sight distribution of X-ray emission in the Galaxy and the density of cosmic rays.

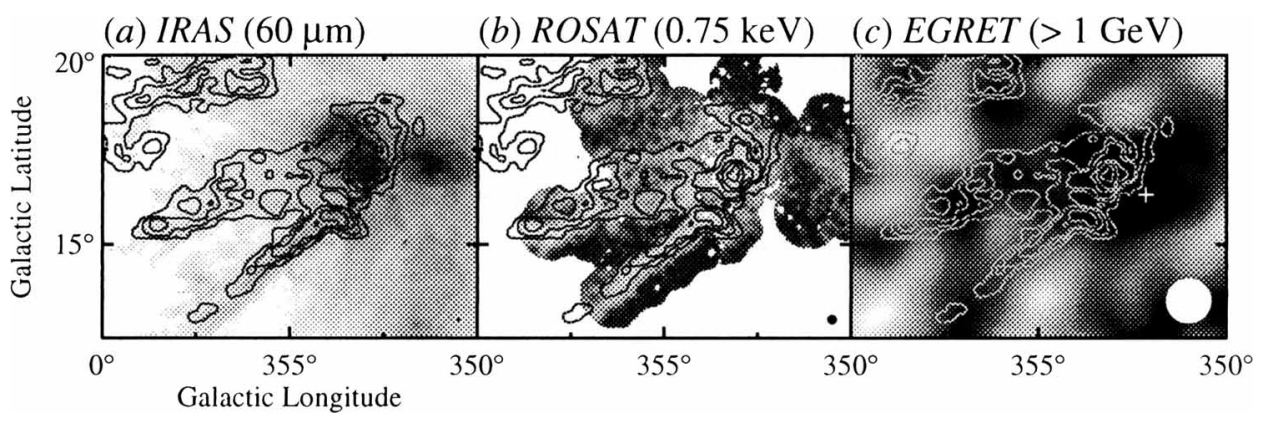

Figure 1. Overlay of contours of integrated CO intensity (de Geus et al. 1990) in the central part of Ophiuchus on images of $(a)$ infrared, $(b)$ X-ray, and $(c)$ high-energy $\gamma$-ray intensity. The infrared emission traces dust heated by stars and star-forming regions, and is well correlated with the $\mathrm{CO}$, which traces dense interstellar gas. The $\mathrm{X}$-ray emission is strongly anticorrelated with the $\mathrm{CO}$, as the interstellar gas and dust absorbs the soft X-ray background. The $\gamma$-ray emission is primarily from $\pi^{0}$ decay after cosmic-ray interactions in the interstellar gas. The white cross marks the position of quasar PKS 1622-253, a $\gamma$-ray source that complicates the analysis. The contour levels are $5,10,15,25,35$, and $45 \mathrm{~K} \mathrm{~km} \mathrm{~s}^{-1}$. Intensity ranges (white-black) are $5-250 \mathrm{M} \mathrm{Jy} \mathrm{sr}^{-1}$ (infrared, logarithmically scaled), (0.4-3) $\times 10^{-5} \mathrm{~s}^{-1} \operatorname{arcmin}^{-2}$ (X-ray), and (0.2-1.8) $\times$ $10^{-5} \mathrm{~cm}^{-2} \mathrm{~s}^{-1} \mathrm{sr}^{-1}(\gamma$-ray). The X-ray image is a mosaic of pointed observations and point sources have been removed.

\section{References}

Burton, W. B. 1985, Astron.Astrophys.Suppl., 62, 36

de Geus, Bronfman, \& Thaddeus 1990, Astron.Astrophys., 231, 137

Digel, S. W., \& Hunter, S. D. 1994, Proc. 2nd Compton Symp., ed. C. E. Fichtel et al. (New York: AIP Press), pp. 484-488

Hauser, M. G. et al. 1995, COBE DIRBE Expl. Supp., Vers. 2.0, COBE Ref. Pub. No. 95-A (Greenbelt, MD: NASA/GSFC)

Hunter, S. D. et al. 1994, ApJ, 436, 216

Snowden, S. L., Digel, S. W. \& Freyberg, M. J. 1994, AAS HEAD meeting, Napa Valley Wheelock, S. L. et al. 1994, IRAS Sky Survey Atlas Expl. Supp., JPL Publ. 94-11 (Pasadena: JPL) 